\title{
P02-09. Dissecting the interference of endotoxins with HIV infection of primary CD4 $\mathrm{T}$ cell
}

\section{A Krarup*, P Rusert and A Trkola}

Address: Institute of Medical Virology, University of Zurich, Zurich, Switzerland

* Corresponding author

from AIDS Vaccine 2009

Paris, France. 19-22 October 2009

Published: 22 October 2009

Retrovirology 2009, 6(Suppl 3):PI4 doi:I0.II86/I742-4690-6-S3-PI4

This abstract is available from: http://www.retrovirology.com/content/6/S3/PI4

(C) 2009 Krarup et al; licensee BioMed Central Ltd.

\section{Background}

Elevated plasma endotoxin levels are considered as indicator of microbial translocation from the gut, and as a potential cause of the systemic immune activation observed in chronic HIV infection. Lipopolysaccharide (LPS) effects on lymphocytes and macrophages have been described to involve amongst other effects, downregulation of CD 4 and CCR 5 and induction of CCL3/MIP-1 $\alpha$ and CCL4/MIP-1 $\beta$ secretion. This alteration in receptor expression could potentially reduce HIV entry and may thus pose a problem for assessing plasma antibody neutralization in patient samples.

\section{Methods}

In the present study we evaluated the influence of LPS on primary CD4 T cells cultivated under various in vitro conditions. CD8-depleted PBMC and unseparated PBMC were subjected to LPS from four different bacterial strains (E. coli 0127:B8, E. coli 0127:B8, P. aeruginosa and S. enterica serotype enteritidis). CD4 and coreceptor levels were measured at various time points after treatment and the infectability of the different cells compared using envelope pseudotyped reporter virus.

\section{Results}

We observed no inhibitory influence of LPS on the infectivity of PBMC by HIV env pseudotyped and MuLV env pseudotyped virus over the entire range of LPS probed (1$1000 \mathrm{pg} / \mathrm{ml}$ LPS). Equally, we observed no shifts in the inhibitory capacity of neutralizing antibodies regardless whether cells were pretreated with LPS, or upon simulta- neous addition of LPS. Equally unaffected by LPS were TZM-bl cells.

\section{Conclusion}

Our data indicate that HIV pseudotype infection and neutralization assays on PBMC and TZM-bl cells are not affected by trace sources of LPS. Plasma endotoxin at levels reported in HIV infection will thus not interfere with detection of neutralizing antibody activity in patient sera. 